\title{
Causes and assessment of subacute and chronic wrist pain
}

\author{
Janice Chin-Yi $\underline{\mathrm{LiaO}}^{1}$, MBBs, MRCs, Alphonsus Khin Sze Chong${ }^{1,2}$, MBBS, FAMs, David Meng Kiat $\underline{\operatorname{Tan}}^{1}$, MBBS, MMed
}

ABSTRACT Wrist pain is a common presentation to the general practitioner and emergency department. Most cases are simple to treat, and pain frequently resolves with conservative treatment. However, there are certain conditions, such as scaphoid nonunion and Kienböck's disease, where delayed diagnosis and treatment can result in long-term deformity or disability. This article covers the various causes of wrist pain, recommendations on how wrist pain should be assessed, as well as details some of the common conditions that warrant specialist referral.

Keywords: chronic wrist pain, physical examination, wrist injuries, wrist pain

\section{INTRODUCTION}

Wrist pain is a common clinical problem. ${ }^{(1)}$ Many acute cases are sprains or contusions following a minor injury, or tendinitis following an episode of overuse. These problems generally resolve within weeks with rest, temporary immobilisation and symptomatic pain relief.

Wrist pain that does not resolve after six weeks to three months is a frequent diagnostic challenge to primary care physicians. The differential diagnoses are extensive due to the complexity of the bony articulations and surrounding soft tissue. Through good history taking and physical examination, followed by the appropriate radiological investigations, a diagnosis is achieved $78 \%$ of the time. ${ }^{(2)}$ A systematic examination helps localise the wrist pain according to radialsided, central and ulnar-sided tenderness, as well as formulate an appropriate diagnosis.

\section{ASSESSMENT OF THE WRIST}

A detailed history, with attention given to key points such as handedness, occupation, hobbies and mechanism of injury, facilitates diagnosis and management according to a patient's expectations and functional requirements. If there is a history of injury, the mechanism, onset and location of pain, aggravating and relieving factors, and previous treatment should be noted. For nontraumatic wrist pain, the clinician should ask the patient whether involvement of other joints and systemic symptoms are present, as this may suggest inflammatory arthritis or degenerative arthritis.

When examining the wrist, the clinician should inspect for any swelling, deformity, muscle wasting or skin changes. Tenderness should also be localised to the radial, central or ulnar side of the wrist. More specific locations, for example, tenderness over the scaphoid tubercle or in the anatomical snuffbox may suggest scaphoid fractures, whereas tenderness in the scapholunate interval or ulnar fossa may indicate scapholunate ligament injuries or triangular fibrocartilage complex (TFCC) injuries, respectively. The wrist should then be assessed through passive and active range of motion exercises. Special tests (e.g. Finkelstein's and Watson's tests) to differentiate specific wrist conditions are described in later sections. Ideally, patients with persistent swelling and tenderness, or a recent or remote history of trauma, should undergo plain radiography to exclude inflammatory arthritis or fractures.

\section{RADIAL-SIDED WRIST PAIN Scaphoid fractures}

Scaphoid fractures are the most common carpal fractures. They are usually sustained from a fall on an outstretched hand. In patients with scaphoid fractures, tenderness may be elicited in the anatomical snuffbox, over the dorsoradial aspect of the wrist or scaphoid tubercle (Fig. 1). Axial compression of the first metacarpal on the scaphoid can also produce pain. Plain radiograph of the wrist should include posteroanterior (PA), lateral and scaphoid views (Fig. 2).

Initial radiograph in the acute phase may not reveal an undisplaced fracture of the scaphoid. If clinical suspicion remains strong, the patient should be treated with two weeks of thumb spica cast or splint, followed by repeat radiography. ${ }^{(3)}$ However, to avoid unnecessary immobilisation in a certain subset of patients, further assessment can be done with other imaging modalities, including bone imaging, computed tomography $(\mathrm{CT})$ and magnetic resonance (MR) imaging.

\section{Scaphoid nonunion}

Most undisplaced fractures heal uneventfully with proper treatment in a cast, while displaced fractures require internal fixation. However, scaphoid nonunion is not uncommon,

${ }^{1}$ Department of Hand and Reconstructive Microsurgery, National University Hospital, ${ }^{2}$ Department of Orthopaedic Surgery, Yong Loo Lin School of Medicine, National University of Singapore, Singapore

Correspondence: Dr Alphonsus Chong, Senior Consultant and Head, Department of Hand and Reconstructive Microsurgery, National University Hospital, IE Kent Ridge Road, NUHS Tower Block, Level 11, Singapore 119228. doscksa@nus.edu.sg 

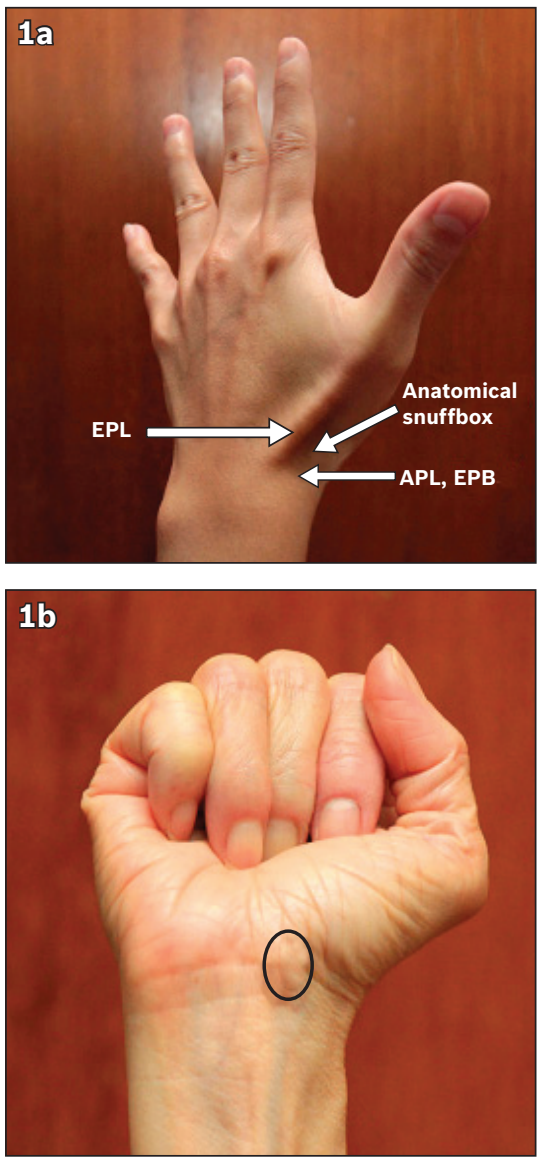

Fig. 1 Tenderness from scaphoid pathology can be palpated (a) in the anatomical snuffbox bounded radially by the abductor pollicis longus ( $A P L$ ) and extensor pollicis brevis (EPB), and ulnarly by the extensor pollicis longus (EPL); and (b) over the scaphoid tubercle (circled), which becomes more prominent on radial deviation of the wrist.

and an incidence of $5 \%-12 \%$ has been reported. ${ }^{(4)}$ Scaphoid nonunion can present as persistent wrist pain or new-onset wrist pain with no recollection of a traumatic event. Therefore, it is important to exclude scaphoid pathology in patients with radial-sided wrist pain. Early recognition and correction of the nonunion reduces the risk of progressive carpal collapse and subsequent osteoarthritis. Displacement of fracture fragments, cyst formation and sclerosis may be seen on plain radiography. CT may reveal the nonunion more clearly, while MR imaging is used to assess suspected avascular necrosis.

\section{Carpometacarpal joint arthritis of the thumb}

The thumb carpometacarpal joint $(\mathrm{CMCJ})$, after the distal interphalangeal joint, is the second most common joint involved in osteoarthritis of the hand. Patients commonly present to primary care practitioners with insidious pain and swelling at the base of the thumb. They may also have decreased pinch and grip strength, and have difficulty with activities such as writing.

Physical examination typically reveals tenderness along the thumb trapeziometacarpal (TM) joint. Dorsoradial prominence of the thumb metacarpal base secondary to subluxation, which is associated with the loss of abduction of the thumb, may also

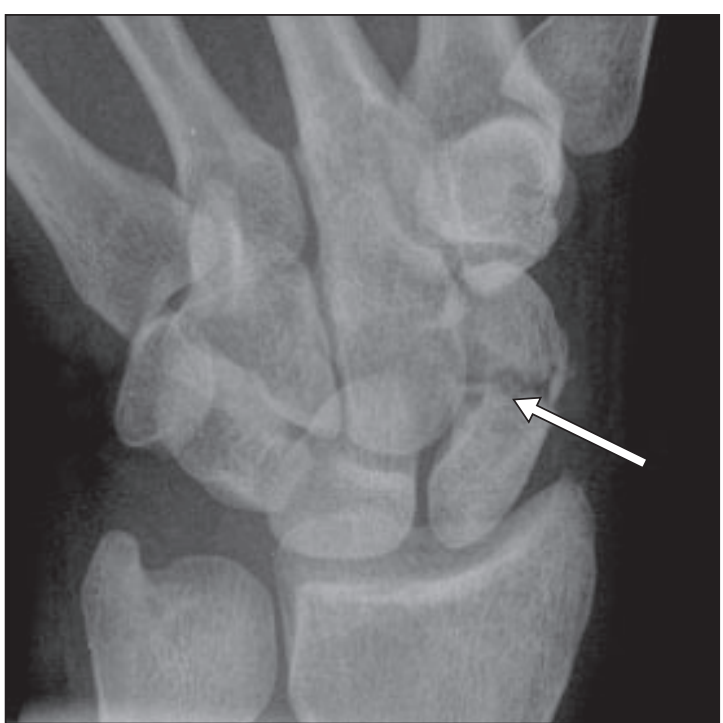

Fig. 2 Plain radiograph of a scaphoid view with a $30^{\circ}$ tube angle that elongates the scaphoid shows scaphoid fracture (arrow).

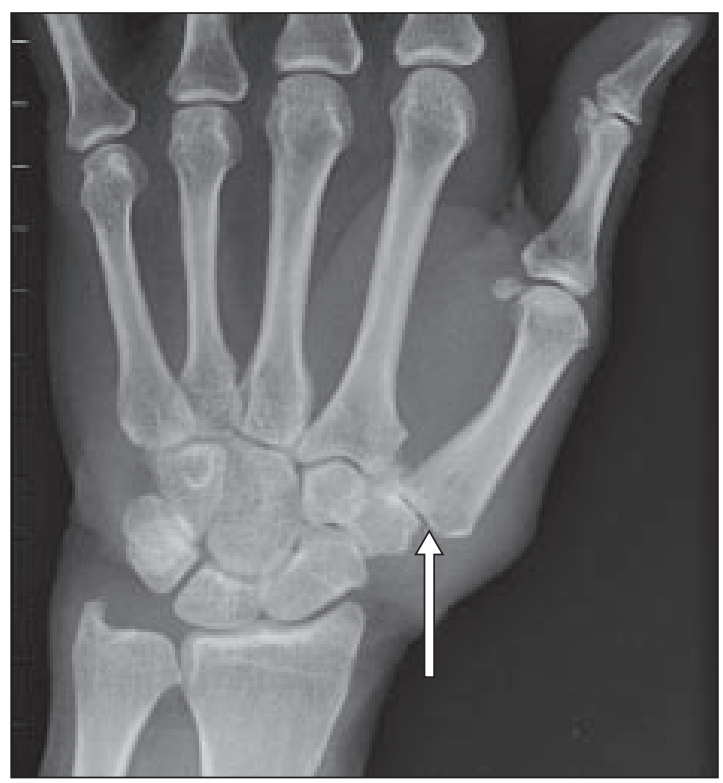

Fig. 3 Plain radiograph shows radial subluxation (arrow) and osteoarthritic changes of the left carpometacarpal joint.

be found. Pain and crepitus that are reproduced with the axial grind test further localise the pathology to the base of the thumb. In addition to the standard views, a stress $30^{\circ} \mathrm{PA}$ view of the $\mathrm{CMCJ}$ helps to assess the degree of joint space loss and subluxation of the TM joint (Fig. 3).

It is important that treatment is based on patient assessment, as most patients with radiographic evidence of first CMCJ arthritis remain asymptomatic. ${ }^{(5)}$ All patients with pain and disability should first be managed conservatively for three to four weeks with activity modification, nonsteroidal antiinflammatory drugs and splinting. The aim is not to completely eliminate pain, but to improve function for activities of daily living, which can be achieved in $60 \%-70 \%$ of patients. ${ }^{(6)}$ Persistent pain and disability, as well as failure of conservative therapy, are indications for excision arthroplasty of the CMCJ, with or without ligament reconstruction. 


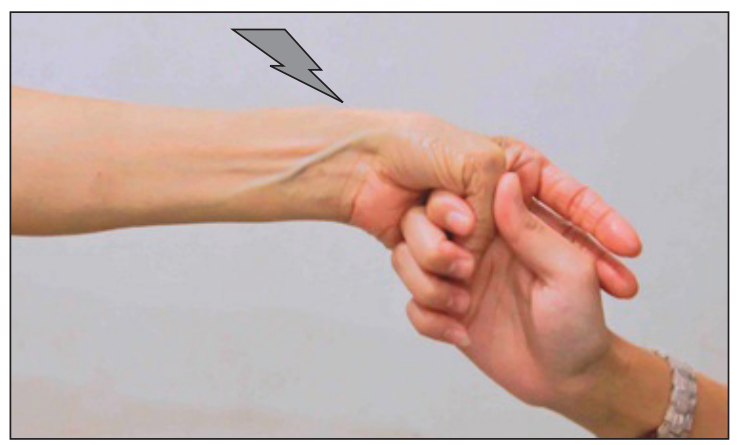

Fig. 4 Positive Finkelstein's test demonstrates pain when the thumb is grasped by the examiner and the wrist is ulnar deviated.

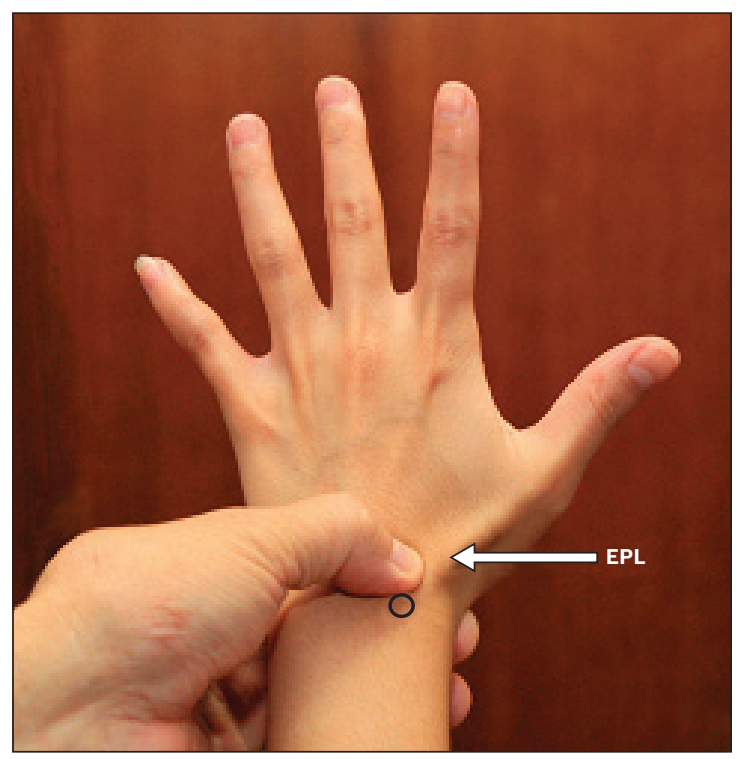

Fig. 5 Photograph shows the scapholunate interval $1 \mathrm{~cm}$ distal to Lister's tubercle (circled). EPL: extensor pollicis longus

\section{de Quervain's tenosynovitis/tenovaginitis}

de Quervain syndrome is a stenosing tenovaginitis of the abductor pollicis longus (APL) and extensor pollicis brevis (EPB) tendons within the first dorsal compartment involved in extension of the thumb.

This condition is frequently diagnosed in females aged 40-50 years. de Quervain syndrome is commonly associated with pregnancy and mothers with infants. Patients present with radial-sided wrist pain that is exacerbated by thumb movement and radiates along the course of the APL and EPB. Localised swelling and tenderness can be palpated over the first dorsal compartment, extending 1-2 $\mathrm{cm}$ proximal to the radial styloid. A positive Finkelstein's test demonstrates pain on ulnar deviation of the wrist (Fig. 4). Differential diagnoses include intersection syndrome, arthritis and scaphoid fracture. Pain from intersection syndrome is usually more proximal, at the junction of the first dorsal compartment and the second dorsal compartment tendons. Arthritis is best appreciated on plain film radiography (Fig. 3). Patients with de Quervain syndrome may be put on a trial of conservative therapy, as described previously. If symptoms are severe or do not improve, steroid injection into the tendon compartment or surgical release of the compartment is recommended.
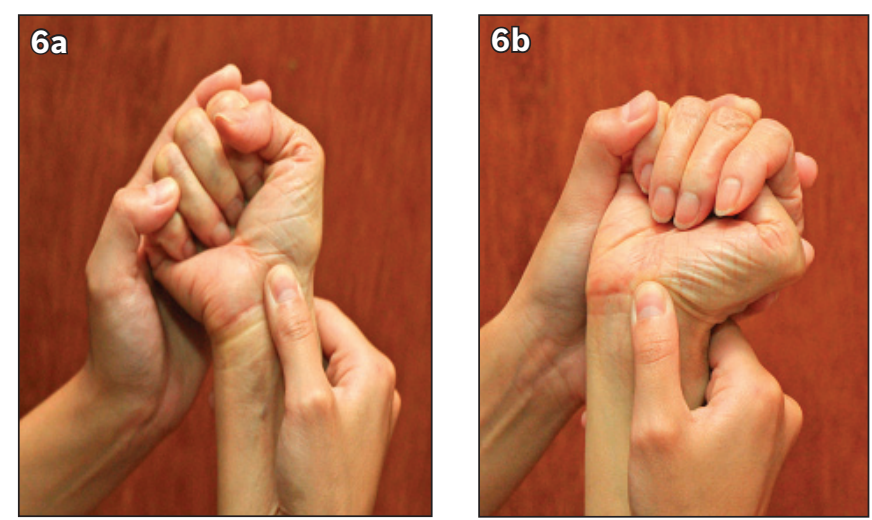

Fig. 6 To perform Watson's test with the patient's wrist in dorsiflexion, place the thumb over the patient's scaphoid tuberosity. While maintaining pressure, the wrist is moved from (a) ulnar deviation to (b) radial deviation. Pain elicited may indicate a fractured scaphoid, or scapholunate instability if a 'clunk' sound is noted when the scaphoid reduces abruptly.

\section{CENTRAL WRIST PAIN Scapholunate instability}

Scapholunate instability is the most common form of ligamentous injury in the wrist resulting from disruption of the mechanical linkage between the scaphoid and lunate. The scapholunate interval is palpated between the third and fourth extensor compartments, approximately $1 \mathrm{~cm}$ distal to Lister's tubercle (Fig. 5).

Early recognition and treatment of scapholunate instability can prevent the onset of carpal collapse and arthritis, and stave off salvage procedures of the wrist. However, diagnosis is often delayed due to its varied presentation. Initially, the condition can present with minimal symptoms that are produced with certain characteristic activities. With the passage of time, swelling and progressive dorsal- or radial-sided wrist pain or weakness on loading of the wrist may appear. There may also be a loud 'clunk' sound on palmar flexion. On examination, an area of swelling and tenderness over the scapholunate joint may be palpated. Pain and instability can be accentuated with provocative manoeuvres such as Watson's scaphoid shift test (Fig. 6).

In an acute setting, radiographic examination may be normal. With a complete tear of the scapholunate ligament and secondary ligamentous stabilisers, scapholunate dissociation may ensue. Features that suggest scapholunate dissociation include a scapholunate gap, cortical 'ring' sign and extension of the lunate (Fig. 7). MR imaging is useful for assessing the ligament when plain film radiographs appear normal and symptoms persist. If all radiological modalities are negative, the gold standard is arthroscopy, which allows for both diagnosis and treatment. In chronic cases, open repair or reconstruction of the ligament is necessary.

\section{Wrist ganglion}

Ganglions are the most common soft tissue tumours of the wrist and hand. They are more prevalent in females and often occur at the age of $20-40$ years. Around $70 \%$ of ganglions 


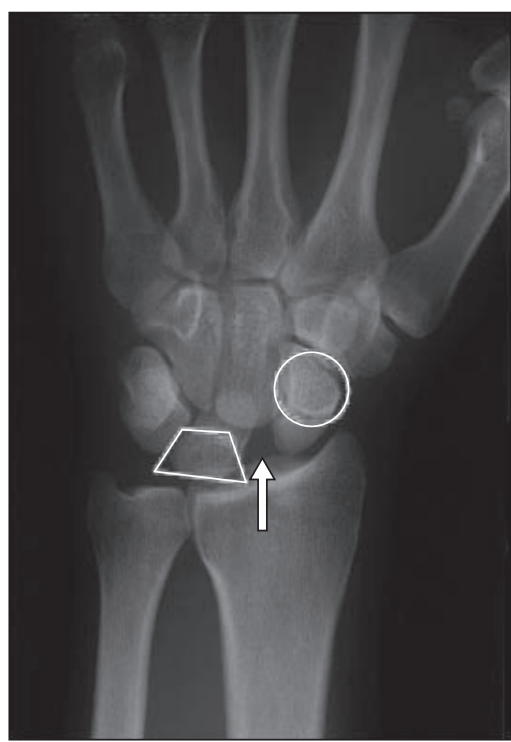

Fig. 7 Plain radiograph of a partially healed distal radius fracture associated with scaphoid fracture and scapholunate dissociation. Signs of scapholunate disruption include increased scapholunate gap (arrow), scaphoid 'ring' sign (circled) due to flexion of the scaphoid with tuberosity superimposed on the waist of the scaphoid, and trapezoid-shaped lunate (trapezoid-shaped outline) due to extension.

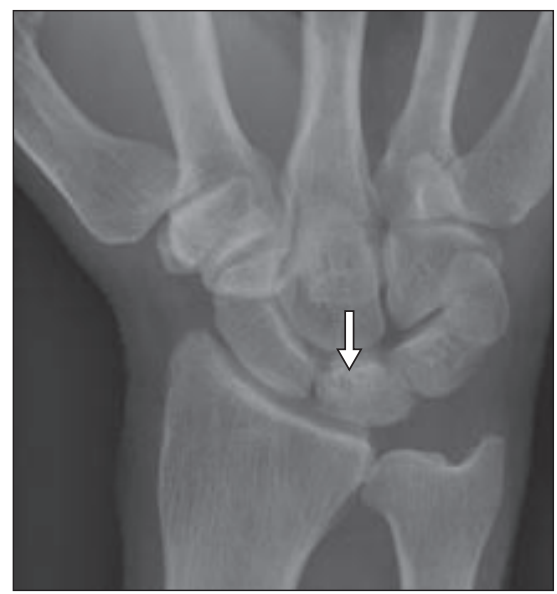

Fig. 8 Posteroanterior radiograph of the wrist of a patient who was diagnosed with stage II Kienböck's disease (on MR imaging) shows increased sclerosis of the lunate (arrow) and negative ulnar variance.

arise from the dorsum of the wrist, especially in proximity to the scapholunate ligament and joint. Patients with dorsal wrist ganglion may present with central wrist pain secondary to mass effect or compression of the posterior interosseous nerve, whereas those with occult ganglion may present with undiagnosed chronic wrist pain.

On examination, a ganglion appears as a smooth subcutaneous mass that transilluminates when a penlight is held next to it. A plain radiograph should be obtained to assess any interosseous involvement and to rule out other differential diagnoses. Ultrasonography can be used to detect occult ganglions or assess atypical cysts. ${ }^{(7)}$ When an occult ganglion is suspected, MR imaging offers an objective display of the occult ganglion and its anatomical relationships.

When a definitive diagnosis of a ganglion is made, it can often be treated conservatively. Rupture or aspiration of a ganglion has a high recurrence rate. If the diagnosis is uncertain or the ganglion is symptomatic, excision biopsy should be considered.

\section{Kienböck's disease}

Kienböck's disease is the most common type of idiopathic carpal avascular necrosis (AVN). Although it is relatively rare, if undiagnosed, patients can develop pain at rest and progressive wrist stiffness, loss of motion and reduced grip strength. Due to the progressive nature of this condition, early diagnosis and referral is crucial.

Patients with Kienböck's disease can present with dorsal wrist pain over the region of the lunate, reactive synovitis and swelling. Patients may also have decreased wrist motion and grip strength. In the early stages, plain radiographs are normal or demonstrate increased radiodensity of the lunate with fracture lines (Fig. 8). As the condition progresses, carpal collapse, scaphoid rotation and height loss, and degenerative arthritic changes will be visible. If radiographs are not diagnostic, MR imaging should be obtained to facilitate early diagnosis and intervention.

Treatment options are typically surgical. Although surgical treatment does not reverse or prevent carpal collapse, it has been shown to retard the process. ${ }^{(8)}$ Conservative treatment with immobilisation may be attempted, but it has not been shown to be effective in later stages of the disease.

\section{ULNAR-SIDED WRIST PAIN Triangular fibrocartilage complex tear}

The TFCC is a major stabiliser of the distal radioulnar joint (DRUJ), absorbing approximately $20 \%$ of the axial load of the wrist. The force transmitted is higher in pronation than supination, and with forced grip. Thus, activities that place the wrist in this position, such as golf, badminton and gymnastics, play a role in TFCC injuries. Patients with TFCC tears often complain of pain over the ulnar aspect of the wrist that is associated with clicking when hyperpronating and supinating, such as when opening jars or doors. Point tenderness can be elicited in the hollow between the pisiform, the flexor carpi ulnaris and ulnar styloid (Fig. 9). The press test, which requires a seated patient to push the body off the chair using the affected wrist, has been recommended with $100 \%$ sensitivity in preoperative tear detection. ${ }^{(9)}$

Although TFCC tears are not visible on plain radiographs, radiography helps to exclude other differential diagnoses such as ulnar styloid fractures and ulnocarpal abutment syndrome. A PA view may also reveal features associated with TFCC tear, including ulnar styloid base avulsion and increased ulnar variance. MR imaging, conventional arthrography and MR arthrography are useful imaging modalities. However, arthroscopy remains superior in revealing TFCC injuries, as it has the advantage of being a therapeutic modality as well. 


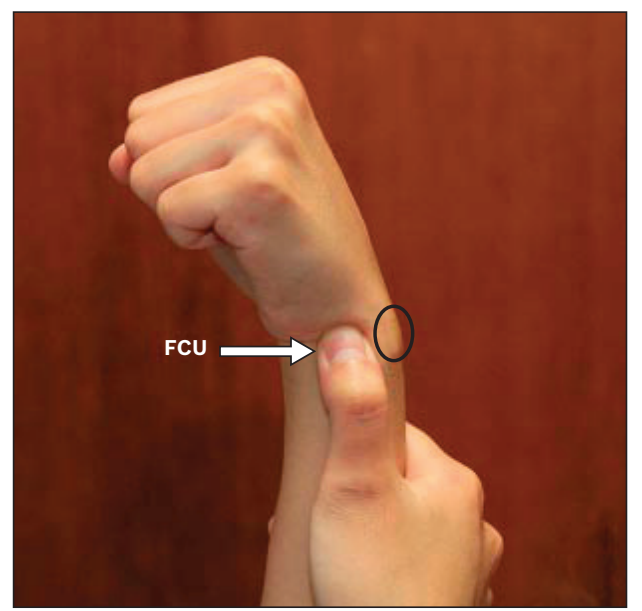

Fig. 9 The ulnar fovea is located under the thumb, between the flexor carpi ulnaris (FCU) and ulna styloid (circled).

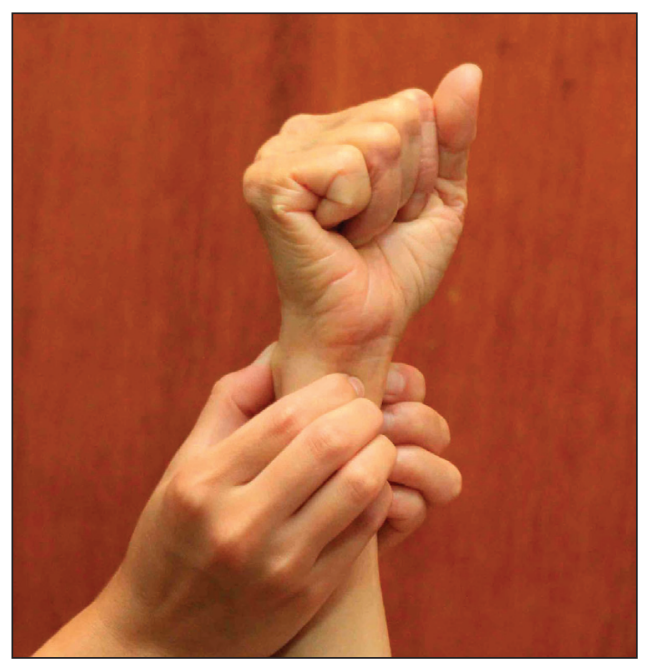

Fig. 10 To perform the distal radioulnar joint shuck test with the patient's forearm in supination, the distal radius is stabilised while the distal ulnar is translated in the lateral plane. A positive test demonstrates laxity, crepitation or grinding of the ulnar head against the distal radius.

\section{Distal radioulnar joint instability}

In patients with DRUJ injuries, a missed diagnosis can result in significant disability. Chronic instability can result from distal radius fracture malunion, ulnar styloid fracture nonunion and DRUJ dislocation. Patients often present with focal pain and decreased forearm rotation. Arthritis needs to be excluded because ligament reconstruction is contraindicated. Arthritis is suggested if pain is produced during the ulnar compression test, where the ulnar head is compressed against the sigmoid notch with a radially directed force. In DRUJ instatbility, the DRUJ shuck test may demonstrate laxity. This is especially significant if laxity is absent in the contralateral wrist (Fig. 10).

The appearance of an isolated DRUJ subluxation on a lateral plain film can be dismissed as positional. It is essential that true lateral views be taken so the palmar cortex of the pisiform bone overlies the central third of the interval between the palmar cortices of the distal scaphoid pole and the capitate head.(10) Other suggestive features of instability include widening of the radioulnar joint on PA view,
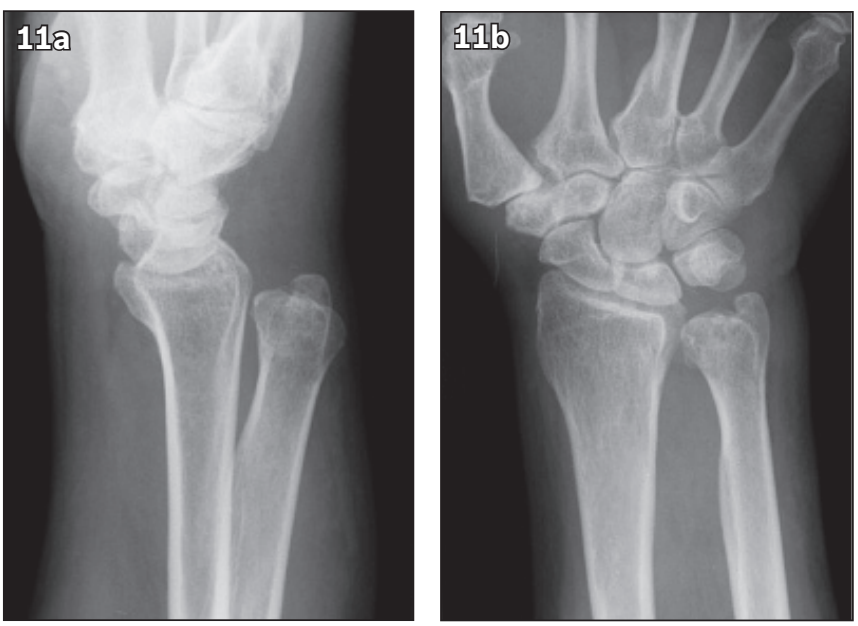

Fig. 11 Plain radiograph in the (a) lateral view shows dorsal dislocation; and (b) posteroanterior view shows widening of the distal radioulnar joint.

fracture at the base of ulnar styloid and shortening of the radius (Fig. 11). If the diagnosis is in question, CT of both wrists in identical rotation is the most useful method to evaluate DRUJ instability and rule out DRUJ arthritis.

\section{Extensor carpi ulnaris pathology}

In terms of frequency of tendonitis about the wrist, external carpi ulnaris (ECU) tendinitis is second only to de Quervain's tenosynovitis. ${ }^{111}$ However, without an accurate clinical evaluation of the patient, poorly localised ulnar-sided wrist pain may not be recognised. Frequently, a history of repetitive ulnar deviation of the wrist from actions such as typing or hammering may be present. The salient features are point tenderness over the sixth dorsal compartment, swelling along the ECU tendon, ulnar-sided wrist pain on passive palmar flexion and radial deviation with forearm supinated, and palpable crepitus with ECU excursion. Temporary or permanent response to a localised injection of an anaesthetic into the sixth compartment is diagnostic in most cases. This may also be therapeutic if combined with a steroid agent such as triamcinolone.

ECU subluxation may be confused with DRUJ or TFCC injuries. ECU is often caused by a sports-associated injury from forceful forearm supination, wrist palmar flexion and ulnar deviation (e.g. while pitching a ball or swinging a racket). The tendon displaces in the ulnar and palmar direction with a painful snapping sensation, and relocates on pronation. MR imaging shows malposition of the tendon and may show inflammation of the tendon sheath. Other disorders of ECU tendon, including tendonitis and tendon rupture, are well assessed by MR imaging.

\section{DIFFUSE WRIST PAIN}

Osteoarthritis and inflammatory arthritis such as rheumatoid arthritis (RA) and systemic lupus erythematous involving the wrist can cause chronic diffuse wrist pain associated with swelling, limited range of motion and weakness. However, 

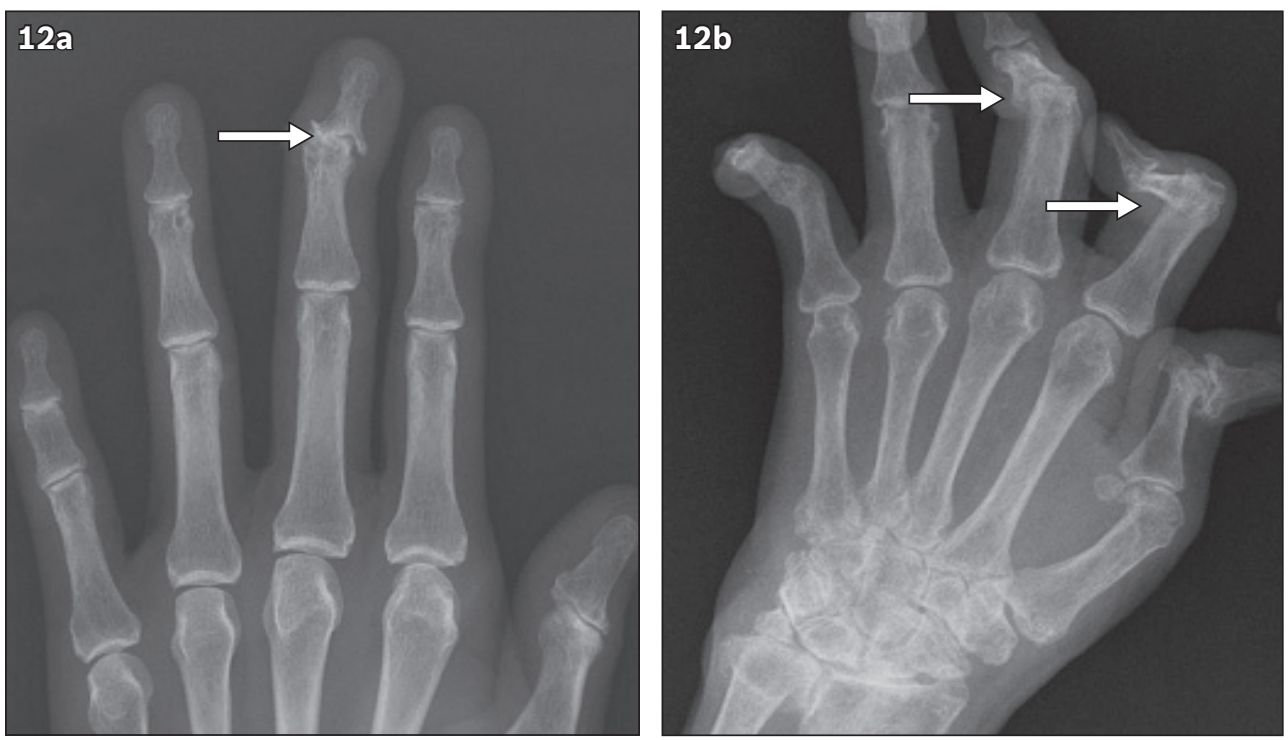

Fig. 12 Radiographs show (a) osteoarthritic changes (arrow) with marginal osteophytes, subchondral sclerosis and narrowed joint spaces in the distal interphalangeal joint of the hand; and (b) rheumatoid arthritis involving the radiocarpal joints, distal radioulnar joints, proximal interphalangeal joint and interphalangeal joint of the thumb. Boutonniere deformity is seen in the index and middle finger (arrows).

crystal arthropathies, which include gout and pseudogout, present with recurrent attacks of acute inflammatory arthritis that is characterised by a red, tender, hot and swollen wrist.

Although primary osteoarthritis has no known cause, it is associated with ageing and overuse. In some patients, it is secondary to pre-existing damage to the wrist joint (e.g. fractures, ligament injuries or Kienböck's disease). Overall, osteoarthritis occurs more frequently in the hand than the wrist, whereas RA commonly affects the wrist and metacarpal phalangeal joint. Other classic features of RA include boutonniere and swan-neck deformities, as well as the inability to move the fingers secondary to tendon rupture.

Diagnosis of osteoarthritis is typically confirmed on plain radiography (Fig. 12a). In early stages of RA, there may be no changes seen on radiography. As the disease progresses, soft tissue swelling, juxta-articular osteopenia and joint space loss may be visible (Fig. 12b). The detection of rheumatoid factors or anti-citrullinated protein antibodies confirms the diagnosis.

Osteoarthritis can be managed primarily by the family physician, while other inflammatory arthritis is worthy of referral to specialist care. The aim of treatment is to relieve pain, prevent deformity and maintain function. Surgical intervention (e.g. arthrodesis and arthroplasty) is indicated when nonsurgical methods fail to control pain or prevent deformities that lead to impaired hand function.

\section{CONCLUSION}

Primary care physicians are often the first to evaluate and treat patients with chronic wrist pain. Tenderness can be localised to specific anatomical structures with special tests, for example, Finkelstein's test and the compression test, to support a specific diagnosis. If the diagnosis remains unclear after conventional radiography, further imaging, including CT, MR imaging and ultrasonography, are indicated. Wrist pain caused by tendinopathies and noninflammatory arthritis are simple to treat and do not require specialised treatment by a hand surgeon. However, most other conditions causing wrist pain warrant a specialist review in order to optimise functional outcome.

\section{REFERENCES}

1. Nichols CM, Cheng C. Update on the evaluation of wrist pain. Mo Med 2006; 103:293-6.

2. van Vugt RM, Bijlsma JW, van Vugt AC. Chronic wrist pain: diagnosis and management. Development and use of a new algorithm. Ann Rheum Dis 1999; 58:665-74.

3. Smith M, Bain GI, Turner PC, Watts AC. Review of imaging of scaphoid fractures. ANZ J Surg 2010; 80:82-90.

4. Pao VS, Chang J. Scaphoid nonunion: diagnosis and treatment. Plast Reconstr Surg 2003; 112:1666-76.

5. Armstrong AL, Hunter JB, Davis TR. The prevalence of degenerative arthritis of the base of the thumb in post-menopausal women. J Hand Surg Br 1994; 19:340-1.

6. Matullo KS, liyas A, Thoder JJ. CMC arthroplasty of the thumb: a review. Hand (NY) 2007; 2:232-9.

7. Chen HS, Chen MY, Lee CY, Kao MJ, Wang TG. Ultrasonographic examination on patients with chronic wrist pain: a retrospective study. Am J Phys Med Rehabil 2007; 86:907-11.

8. Salmon J, Stanley JK, Trail IA. Kienböck's disease: conservative management versus radial shortening. J Bone Joint Surg Br 2000; 82:820-3.

9. Lester B, Hallbrecht J, Levy IM, Gaudinez R. "Press test" for office diagnosis of triangular fibrocartilage complex tears of the wrist. Ann Plast Surg 1995; 35:41-5.

10. Yang Z, Mann FA, Gilula LA, Haerr C, Larsen CF. Scaphopisocapitate alignment: criterion to establish a neutral lateral view of the wrist. Radiology 1997; 205:865-9.

11. Wood MB, Dobyns JH. Sports-related extraarticular wrist syndromes. Clin Orthop Relat Res 1986; (202):93-102. 


\section{SINGAPORE MEDICAL COUNCIL CATEGORY 3B CME PROGRAMME (Code SMJ 201310B)}

Question 1. Regarding scaphoid fractures:

(a) They are the most common carpal fractures.

(b) The hallmark sign is tenderness in the anatomical snuffbox.

(c) No further treatment is required if the initial radiograph shows no fracture.

(d) Nonunion and avascular necrosis are rare.

Question 2. Regarding de Quervain syndrome:

(a) The extensor pollicis longus and extensor pollicis brevis within the first dorsal compartment are involved.

(b) The axial grind test demonstrates pain on ulnar deviation of the wrist.

(c) Radiography helps to differentiate it from arthritis.

(d) If conservative management fails, the patient should be referred for steroid injection or surgical release of the compartment.

Question 3. Regarding scapholunate instability:

(a) The scapholunate interval is between the third and fourth extensor compartment, approximately $1 \mathrm{~cm}$ distal to Lister's tubercle.

(b) There may be a loud 'clunk' on wrist extension.

(c) Radiographic features include an increased scapholunate gap, a scaphoid 'ring' sign and extension of lunate.

(d) Arthroscopy is the gold standard diagnostic test.

Question 4. Regarding triangular fibrocartilage complex (TFCC) tears:

(a) The TFCC is a major stabiliser of the distal radioulnar joint, absorbing $20 \%$ of the axial load of the wrist.

(b) Injuries often occur during activities that require a forced grip in supination.

(c) The press test is highly sensitive in detecting a TFCC tear.

(d) The Finklestein's test demonstrates tenderness in the ulnar fovea.

Question 5. Regarding extensor carpi ulnaris (ECU) pathology:

(a) ECU tendinitis is the most common tendinitis of the wrist.

(b) ECU tendinitis causes ulnar-sided wrist pain on palmar flexion and radial deviation with the forearm supinated.

(c) The mechanism of injury for ECU subluxation is forceful forearm supination, wrist flexion and radial deviation.

(d) In ECU subluxation, the tendon displaces in the ulnar and palmar direction with a painful snapping sensation, and relocates on pronation.

\section{Doctor's particulars:}

Name in full

MCR number

Specialty:

Email address

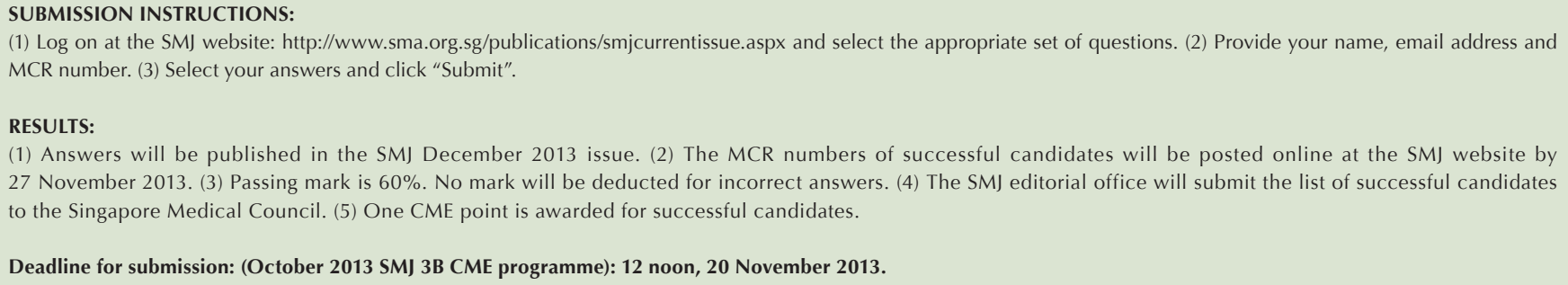

(1) Log on at the SMJ website: http://www.sma.org.sg/publications/smjcurrentissue.aspx and select the appropriate set of questions. (2) Provide your name, email address and MCR number. (3) Select your answers and click "Submit".

RESULTS:

(1) Answers will be published in the SMJ December 2013 issue. (2) The MCR numbers of successful candidates will be posted online at the SMJ website by 27 November 2013. (3) Passing mark is $60 \%$. No mark will be deducted for incorrect answers. (4) The SMJ editorial office will submit the list of successful candidates to the Singapore Medical Council. (5) One CME point is awarded for successful candidates.

Deadline for submission: (October 2013 SMJ 3B CME programme): 12 noon, 20 November 2013. 